\title{
Interaction between \\ Ultraviolet Light and $\gamma$-Radiation Damage in the Induction of Mutants of Escherichia coli: the Effect of Some Modifying Treatments
}

\author{
By B. A. BRIDGES AND R. J. MUNSON \\ Medical Research Council Radiobiological Research Unit, \\ Harwell, Didcot, Berkshire \\ AND C. F. ARLETT AND D. R. DAVIES \\ Wantage Research Laboratory (A.E.R.E.), Wantage, Berkshire
}

(Accepted for publication 5 September 1966)

\begin{abstract}
SUMMARY
The effect of various modifying agents has been studied on the synergistic interaction of u.v.- and $\gamma$-radiation in the induction of prototrophic revertants of tryptophan-requiring Escherichia coli $\mathrm{B} / \mathrm{r}$ WP 2 . The component of $\gamma$-radiation damage involved in the interaction shows a large effect of oxygen during irradiation (dose modifying factor of up to 3 ) and is thus more like lethal than mutational damage. The interaction mutants respond to various postirradiation treatments in the same way as u.v.-induced, but not $\gamma$-induced, mutants, i.e. they are photoreactivable, their frequency is increased by acriflavine and nutrient broth after irradiation and they are susceptible to mutation frequency decline in minimal medium. It is postulated that damage induced in DNA by $\gamma$-radiation inhibits the repair of premutational lesions induced by u.v. thus increasing the yield of u.v.-induced mutants. The possible nature of the system involved in this repair (which is not that responsible for the excision of thymine dimers from DNA) is discussed.
\end{abstract}

\section{INTRODUCTION}

In the preceding paper (Davies, Arlett, Munson \& Bridges, 1967) we have shown that more prototrophic revertants of the tryptophan requiring strain Escherichia coli wP 2 are induced by combined treatments with ultraviolet light (u.v.) and $\gamma$-radiation than would be expected if the effect of the treatments were merely additive. The additional mutants, which we have termed interaction mutants, have been shown to arise by the interaction of u.v.- and $\gamma$-radiation damage during post-irradiation development. For the purpose of this paper the frequency of interaction mutants per survivor is defined as the number of mutants per survivor induced by a combined u.v. $-\gamma$ treatment less the number of mutants per survivor induced by each treatment given separately to similar bacterial suspensions. In this paper we record the effect on the yield of interaction mutants of various modifying treatments which are known to affect either the initial amount of $\gamma$-radiation damage (e.g. oxygen) or the cellular repair processes affecting u.v. damage (e.g. photoreactivating light, acriflavine, nutrient broth). The results indicate that the interaction mutants behave as though they are u.v.-induced. 


\section{METHODS}

Escherichia coli $\mathrm{B} / \mathrm{r}$ WP 2 was used for most experiments; where stated confirmatory


dark repair processes.

Microbiological techniques and details of the irradiation sources have been given elsewhere (Davies et al. 1967). Glucose-salts medium enriched with $0.75 \mu \mathrm{g} . / \mathrm{ml}$. tryptophan and solidified with $1.5 \%$ agar was used for all experiments except those involving mutation frequency decline when enrichment was made with $2.5 \%$ Oxoid nutrient broth instead of tryptophan.

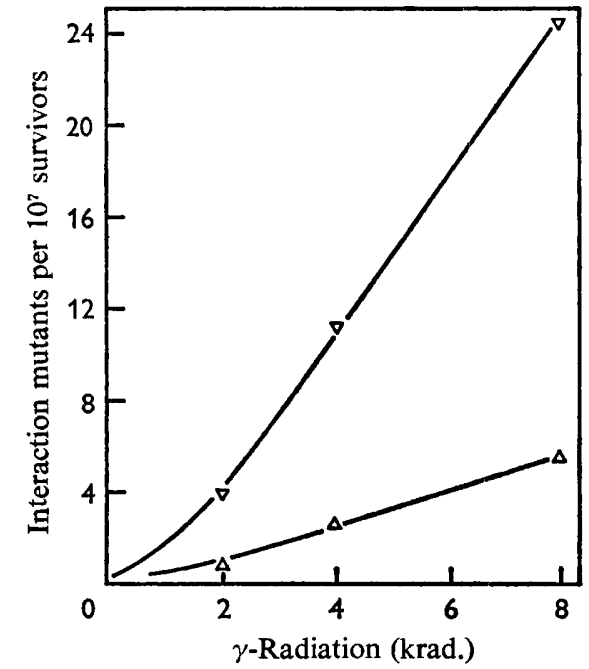

Fig. 1

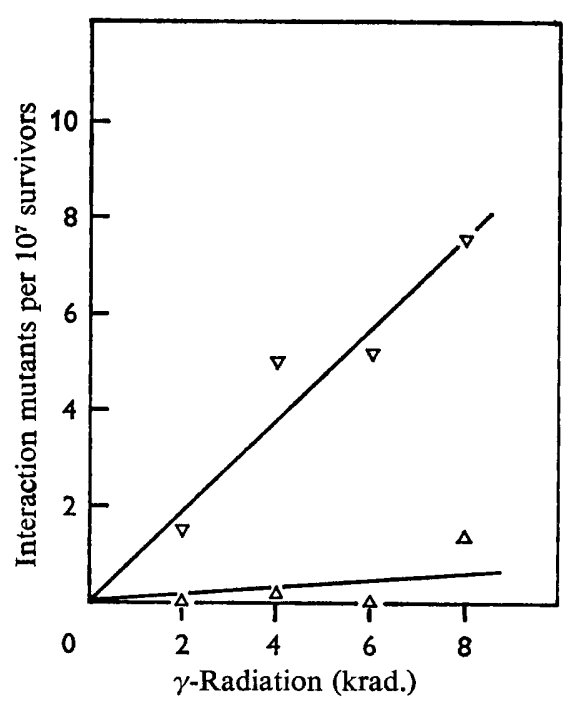

Fig. 2

Fig. 1. Effect of presence $(\nabla)$ or absence $(\triangle)$ of oxygen during $\gamma$-irradiation on the production of interaction mutants after 67.5 ergs $\mathrm{mm}^{-2}$ u.v.

Fig. 2. Effect of photoreactivation after 45 ergs mm. -2 $^{-2}$ u.v. on subsequent yield of interaction mutants with $\gamma$-radiation. $\nabla$, no photoreactivation; $\Delta$, with photoreactivation.

The source of photoreactivating light was a $500 \mathrm{~W}$ photofiood lamp. Suspensions of bacteria in the glucose-salts medium were placed for $20 \mathrm{~min}$. at $10 \mathrm{~cm}$. from the lamp in a water-bath maintained at $15^{\circ}$. Non-photoreactivated controls were wrapped in aluminium foil during an identical treatment.

Acriflavine was obtained from General Biochemicals.

\section{RESULTS}

\section{Effect of oxygen}

When a dose of u.v. was followed by a range of $\gamma$ doses given with either oxygen or 'oxygen-free' nitrogen bubbling through the suspension, interaction mutants in Escherichia coli $\mathrm{B} / \mathrm{r}$ WP 2 were induced as shown in Fig. 1. The slope of the response curve under oxygenated conditions varied from 1.5 to 3 times that under anoxic conditions in four different experiments. 


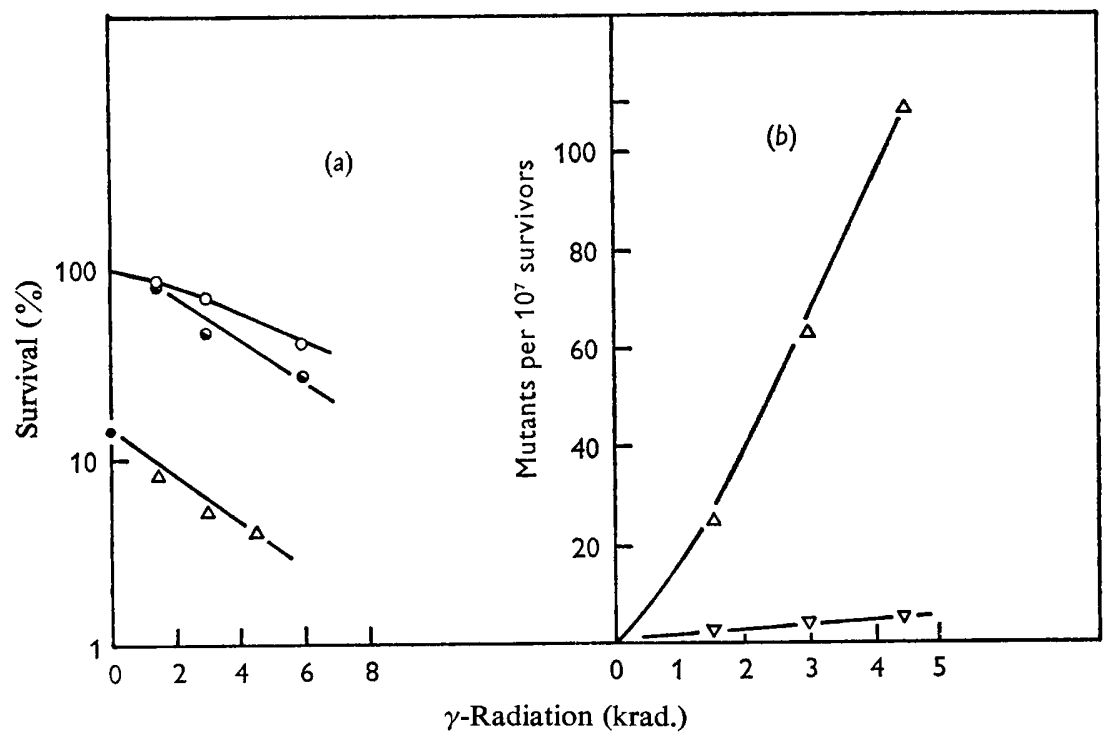

Fig. 3. (a) Effect of $3 \mu \mathrm{g}$./ml. acriflavine in the plating medium and u.v. on the lethal action of $\gamma$-radiation on Escherichia coli. $\bigcirc$, no acriflavine; $\ominus$, acriflavine; $\ominus, 67.5$ ergs mm. ${ }^{-2}$ u.v. on acriflavine plates; $\triangle, 67 \cdot 5$ ergs mm..$^{-2}$ u.v. followed by $\gamma$-radiation, on acriflavine plates. (b) Yield of interaction mutants by $67.5 \mathrm{ergs} \mathrm{mm} .^{-2}$ u.v. followed by a range of doses of $\gamma$-radiation. $\nabla$, no acriflavine; $\Delta, 3 \mu \mathrm{g} . / \mathrm{ml}$. acriflavine in plating medium.

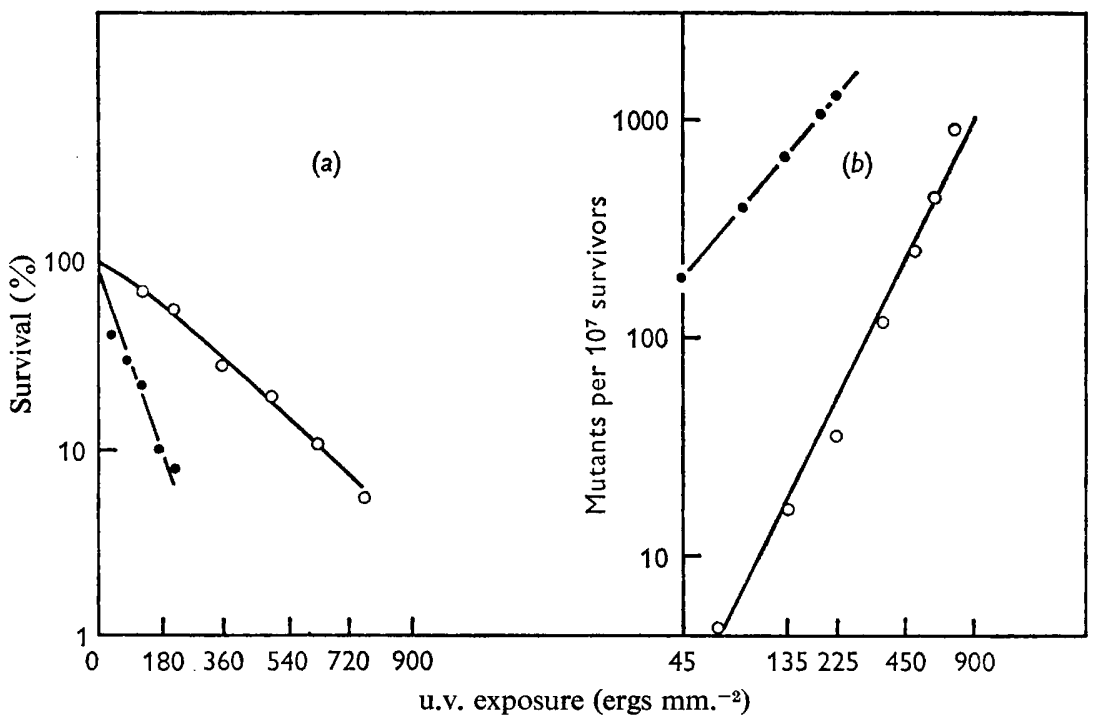

Fig. 4. (a) Lethal effect of u.v. on Escherichia coli plated on medium with ( $)$ and without (O) $3 \mu \mathrm{g} . / \mathrm{ml}$. acriflavine. (b) Yield of u.v.-induced mutants plated on medium with (O) and without (O) $3 \mu \mathrm{g} . / \mathrm{ml}$. acriflavine. 


\section{Effect of photoreactivating light}

When a photoreactivating treatment was given between a u.v. dose and a $\gamma$-radiation dose, the rate of induction of interaction mutants in Escherichia coli $\mathrm{B} / \mathrm{r}$ WP 2 was reduced by a factor of about 8 (Fig. 2). This also happened with $E$. coli WP 2 hcr- $^{-}$ where, in addition, it was shown that the photoreactivating treatment was effective even when given after the $\gamma$-irradiation.

\section{Effect of acriflavine}

The addition of acriflavine to the plating medium is known to enhance the lethal and mutagenic action of u.v. (Witkin, 1963) but not the mutagenic action of $\gamma$ radiation (Bridges \& Munson, 1964). Our experiments, using $3 \mu \mathrm{g} . / \mathrm{ml}$. acriflavine,



Fig. 5. Decline of mutation frequency with time as revealed on broth-enriched plates when bacteria are held in glucose salts medium at $37^{\circ}$ after irradiation with $195 \mathrm{ergs} \mathrm{mm} .^{-2}$ and/or 4 krad. $\gamma$-radiation. $O$, total induced mutants after u.v. and $\gamma$-irradiation; $\Delta$, total induced mutants after u.v. and $\gamma$-irradiation less those induced by $\gamma$-radiation alone; $\bullet$, mutants induced by u.v. alone; $\nabla$, interaction mutants, i.e. total mutants induced by combined u.v.and $\gamma$-irradiation less those induced by u.v. and $\gamma$-radiation alone. In ( $a$ ) absolute mutation frequencies are plotted, in $(b)$ these have been plotted as a percentage of their values immediately after irradiation.

confirm this and also shown an enhancement of the lethal action of $\gamma$-radiation (Fig. 3a) similar to that observed by Alper (1963) with $E$. coli B/H. The induction of interaction mutants was enhanced by acriflavine (Fig. $3 b$ ) to roughly the same extent as the induction of u.v. mutants (Fig. 4).

\section{Mutation frequency decline}

It is a well-established fact, dating from the observations of Witkin (1956), that more u.v.-induced mutations appear on glucose + salts plates supplemented with a little nutrient broth than on plates supplemented with tryptophan alone. The amino acids in the broth in some way stabilize the premutational lesions so that more of the latter 
are 'fixed'. If u.v.-exposed bacteria are incubated at $37^{\circ}$ in minimal medium before plating on plates enriched with broth, the number of mutants appearing rapidly falls until by about $40 \mathrm{~min}$. it is the same as that on minimal plates supplemented with tryptophan alone. This process has been termed 'mutation frequency decline' (Doudney \& Haas, 1958); it does not occur with mutations induced by ionizing radiation which are unaffected by the presence of broth (Kada, Brun \& Marcovich, 1960; Munson \& Bridges, 1966). In Fig. $5 a$ we show mutation frequency decline of all the mutants induced by a combined u.v. and subsequent $\gamma$-radiation treatment, of u.v. mutants alone, and of interaction mutants. It is clear that interaction mutants are stabilized on plates enriched with nutrient broth immediately after irradiation, and in the absence of broth show the phenomenon of mutation frequency decline. In Fig. $5 b$ curves for u.v. mutants, interaction mutants, and u.v. and interaction mutants together, have been re-plotted as a percentage of their value at zero time. Neither the rates of mutation frequency decline nor the ratios of final to initial frequencies are significantly different for the three curves.

\section{DISCUSSION}

The oxygen effect experiments may throw some light on the component of $\gamma$ radiation damage which is involved in this interaction between u.v. and ionizing radiation damage. The oxygen enhancement ratio for lethality under the conditions of these experiments is about 3 and for mutation induction about 1.3 (Bridges \& Munson, 1964). For the production of interaction mutants it varied between 1.5 and 3 which might indicate that straightforward $\gamma$-radiation-induced mutations are not involved in the interaction but possibly the same sort of damage which is involved in lethality.

The fact that the u.v. component involved in interaction is photoreactivable does not necessarily imply that thymine dimers are involved since Witkin (1964) has shown that u.v.-mutagenesis may be photoreactivable even in strains known to lack the photoreactivating (dimer-splitting) enzyme. In such a case photoreactivation appears to involve the same indirect mechanism (similar to that involved in photo-protection) which can remove lethal damage (probably including thymine dimers) under certain conditions (Kondo \& Jagger, 1966; Kondo \& Kato, 1966). The sensitivity of the u.v.-interaction component to photoreactivation after both the u.v. and $\gamma$ doses have been given is, of course, a further demonstration that interaction occurs during the post-irradiation period, as is also the post-irradiation sensitivity to acriflavine. The enhancing effect of acriflavine on u.v. mutagenesis is believed to be due to an inhibition of excision-repair and/or mutation frequency decline and the enhanced yield of interaction mutants on acriflavine plates confirms the conclusion of Davies et al. (1967) that the u.v.-interaction component is also sensitive to these processes.

A direct demonstration that interaction mutants are subject to mutation frequency decline (and thus behave like mutants induced by u.v. alone but not $\gamma$-radiation alone) comes from the experiment shown in Fig. 5. It is clear that the proportion of interaction mutants to u.v.-induced mutants is the same in samples plated immediately after irradiation on plates enriched with nutrient broth (which provides partial stabilization against mutation frequency decline) as on those plated after $40 \mathrm{~min}$. in minimal medium (when mutation frequency decline is complete). In addition the rate of decline is the same for interaction as for u.v.-induced mutants. 
The slope of a mutation frequency decline curve depends both on the rates of removal and of fixation of premutational damage. The experiment shown in Fig. 5 makes it unlikely that interaction mutants result from the interference by $\gamma$-radiation with the rates of either of these processes.

So far then it appears that the $\gamma$-component of the damage involved in interaction may be more like lethal than mutational damage, and that the u.v.-component behaves like u.v. mutational damage. The most reasonable interpretation of these results is that damage induced in DNA by $\gamma$-radiation inhibits the repair of premutational lesions induced by u.v.

It is not possible at present to state the precise nature of the $\gamma$-radiation component of damage. There is evidence that both Escherichia coli $\mathrm{B} / \mathrm{r}$ WP 2 and $E$. coli WP 2 hcr $^{-}$ can repair a large proportion of lethal ionizing radiation damage, including possibly single strand breaks (Bridges \& Munson, 1966, McGrath \& Williams, 1966), but it is not known whether the lesions which are ultimately lethal are unrepaired single strand breaks or of a further unidentified type.

There is general agreement that pyrimidine dimers are involved in the induction of mutations by u.v. alone, whether as the sole type of lesion (Hill, 1965; Kondo \& Kato, 1966) or in conjunction with further unidentified lesions (Witkin, 1964, 1966; Doudney, 1963, 1966; Bridges, 1966). One of the strongest arguments against pyrimidine dimers being the only lesions involved is the fact that the removal of premutational lesions by the mutation frequency decline system is severely inhibited by the presence of nutrient broth, whereas the removal of thymine dimers is stimulated (Setlow \& Carrier, 1964).

If we are to explain our mutation interaction results in a way similar to that used by Haynes (1964) to explain his lethal interaction results, we must do so in terms of a repair system able to remove premutational lesions induced by u.v. and inhibited by $\gamma$-radiation lesions (which might imply an ability to repair certain $\gamma$-radiation lesions). So far two candidates appear as possible contenders for the role of this hypothetical repair system.

The first has been described in terms of a low temperature mutation loss (1.t.m.l.). When minimal plates enriched with tryptophan are incubated at temperatures below about $16^{\circ}$ after irradiation, fewer mutants appear compared with plates incubated at $37^{\circ}$, whether after $\gamma$-radiation (Bridges \& Munson, 1964) or u.v. (Munson \& Bridges, 1966).

While this 1.t.m.l. might seem at first sight unlikely to be associated with u.v.- $\gamma$ interaction at $37^{\circ}$, closer examination shows that this need not be so. The phenomenon of 1.t.m.l. has been ascribed to a differential temperature effect on the rates of repair and fixation of premutational lesions (Bridges \& Munson, 1964), but since at temperatures above about $22^{\circ}$ the rates of both repair and fixation apparently share the same dependence upon temperature it follows that the repair may still go on to an appreciable extent at $37^{\circ}$ although we have no means of detecting it. L.T.M.L. is also a suitable candidate for the interaction effect as it occurs in both $\mathrm{hcr}^{+}$and hor- strains to a similar extent and does not appear to be related to excision repair or mutation frequency decline.

The second presumed repair system for u.v. damage (K-reactivation) has been described by Kneser $(1965,1966)$ as being present in wild-type strains of Escherichia coli $\mathrm{K} 12$ and B/r, absent in 'recombination-less' $\mathrm{K} 12$ and inhibited in $E$. coli $\mathrm{B}$ and 
Bs-l. Since it is present in both the hor ${ }^{+}$and hcr- $^{-}$strains which we have used we cannot tell whether it is involved in our interaction phenomenon and, although there is evidence that it operates on both u.v. and $\gamma$-radiation lethal damage, it may act on nongenetic components of radiation damage. In some early work, Demerec \& Latarjet (1946) showed that the rate of mutation induction to phage resistance was the same in $E$. coli $\mathrm{B}$ and $\mathrm{B} / \mathrm{r}$ which indicates that the premutational damage involved there was not amenable to K-reactivation. The same conclusion has been reached by Dr E. M. Witkin (personal communication) for reversion to prototrophy. It is possible that $\mathrm{K}$ reactivation is the system involved in the u.v.- $\gamma$ lethal interaction observed by Haynes (1966) since a similar interaction occurs with $E$. coli WP 2 hcr $^{-}$which possesses Kreactivation (Bridges \& Munson, unpublished observation) but not E. coli Bs-1 (Haynes, 1964 ) which does not. It would obviously be desirable to examine whether synergistic interaction for mutation induction is also absent in $E$. coli Bs-1, as this would give an indication of the possible role of K-reactivation in the mutant interaction phenomenon.

If, however, neither 1.t.m.l. nor K-reactivation is involved in interaction of mutational damage, it would presumably be necessary to postulate a further repair system.

We thank Mr J. Law, Miss R. Dennis and Mrs J. Potter for technical assistance.

\section{REFERENCES}

AlPER, T. (1963). Effects on irradiated micro-organisms of growth in the presence of acriflavine. Nature, Lond. $200,534$.

BRIDGES, B. A. (1966). A note on the mechanism of u.v. mutagenesis. Mutation Res. 3, 273.

Bridges, B. A. \& Munson, R. J. (1964). Temperature, time and X-ray mutagenesis in E. coli. Mutation Res. 1, 362.

Bridges, B. A. \& Munson, R. J. (1966). Excision-repair of DNA damage in an auxotrophic strain of Escherichia coli. Biochem. biophys. Res. Commun. 22, 268.

Davies, D. R., Arlett, C. F., Munson, R. J. \& Bridges, B. A. (1967). Interaction between ultraviolet light and $\gamma$-radiation damage in the induction of mutants of Escherichia coli: the response in strains with normal and reduced ability to repair ultraviolet damage.

Demerec, M. \& Latarjet, M. (1946). Mutations in bacteria induced by radiations. Cold Spr. Harb. Symp. quant. Biol. 11, 38.

DoudNey, C. O. (1963). Ultraviolet light-induced mutation as an event in the physiology of the bacterial cell. In Repair from Genetic Radiation, p. 125. London: Pergamon Press.

DoudneY, C. O. (1966). Photoreversal of ultraviolet light induced mutation, lethality and damage to nucleic acid formation in bacteria. Mutation Res. 3, 280.

Doudney, C. O. \& HAAS, F. L. (1958). Modification of ultraviolet-induced mutation frequency and survival in bacteria by post-irradiation treatment. Proc. natn. Acad. Sci. U.S.A. 44, 390.

HAYNES, R. H. (1964). Molecular localization of radiation damage relevant to bacterial inactivation. In Physical Processes in Radiation Biology, p. 51. New York; Academic Press.

HiLl, R. F. (1965). Ultraviolet induced lethality and reversion to prototrophy in Escherichia coli strains with normal and reduced dark repair ability. Photochem. Photobiol. 4, 563.

Kada, T., Brun, E. \& Marcovich, H. (1960). Comparaison de l'induction de mutants prototrophes par les rayons X et U.V. chez Escherichia coli $\mathrm{B} / \mathrm{r}$ try-. Ann. Inst. Pasteur 99, 547.

KNESER, H. (1965). Dark and K-reactivation in U.V. irradiated Escherichia coli. Z. VererbLehre 97, 102.

KNESER, H. (1966). Absence of K-reactivation in a recombination deficient mutant of E. coli. Biochem. biophys. Res. Commun. 22, 383.

KONDO, S. \& JAGGER, J. (1966). Action spectra for photoreactivation of mutation to prototrophy in strains of Escherichia coli possessing and lacking photoreactivating-enzyme activity. Photochem. Photobiol. 5, 189.

Kondo, S. \& KATO, T. (1966). Action spectra for photoreactivation of killing and mutation to prototrophy in U.V.-sensitive strains of Escherichia coli possessing and lacking photoreactivating enzyme. Photochem. Photobiol. (in the Press).

McGrath, R. A. \& Williams, R. W. (1966). Evidence for repair in X-irradiated DNA. Abstr. Biophys. Soc. p. 73. 
Munson, R. J. \& BRIDGES, B. A. (1966). Non-photoreactivating repair of mutational lesions induced by ultraviolet and ionizing radiations in Escherichia coli. Mutation Res. 3, 461.

SeTLOW, R. B. \& CARRIER, W. L. (1964). The disappearance of thymine dimers from DNA: an error correcting mechanism. Proc. natn. Acad. Sci., U.S.A. 51, 226.

WITKIN, E. M. (1956). Time temperature and protein synthesis: a study of ultraviolet induced mutation in bacteria. Cold Spr. Harb. Symp. quant. Biol. 21, 123.

WITKIN, E. M. (1963). The effect of acriflavine on photoreversal of lethal and mutagenic damage produced in bacteria by ultraviolet light. Proc. natn. Acad. Sci., U.S.A. 50, 425.

WrTKIN, E. M. (1964). Photoreversal and 'dark repair' of mutations to prototrophy induced by ultraviolet light in photoreactivable and non-photoreactivable strains of Escherichia coli. Mutation Res. 1, 22.

WITKIN, E. M. (1966). Radiation-induced mutations and their repair. Science, 152, 1345.

Note added in proof

An interaction between u.v. and X-irradiation has recently been reported by Kada, Doudney \& Haas (1966) (Mutation Res. 3, 118) in E. coli $\mathrm{B} / \mathrm{r}$ WP 2. Interaction occurred only when there was a period of incubation at $37^{\circ}$ between the two irradiations and appeared to depend on RNA and protein synthesis. Furthermore, minimal plates without enrichment were used so that most mutants (both u.v. and X-ray induced) would not be expressed. We consider that the interaction observed under these conditions is probably concerned with the expression of mutations in the absence of tryptophan and is a quite different phenomenon from that which we have examined. 